\title{
Suggestions for further reading
}

History of Mathematics and general reading

[Cou], [Dav], [Kli], [Kri], [Ste]

Set Theory

[End], [Hal], [Sto], [Sup]

Real Analysis

[Apo], [Bar], [Ber], [Boa], [Gau]

Metric spaces

[Cop-1], [Gof]

Complex Analysis

[Ahl], [Con], [Pon], [Rem], [Rud]

Algebra

[Art], [Fra], [Lang] 
This page is intentionally left blank 


\section{Symbol Index}

\section{Chapter 1}

$\begin{array}{ll}/ & : \text { not, } 9 \\ \Rightarrow & : \text { implies, } 9 \\ \Leftrightarrow & : \text { if and only if, iff, } 9 \\ \wedge & : \text { and, } 9 \\ \vee & : \text { or, } 9 \\ \forall & : \text { for all, } 9 \\ \exists & : \text { there exists, } 9 \\ \exists ! & : \text { there exists exactly one, } 9 \\ \epsilon & : \text { member of, } 9 \\ = & : \text { equal, } 9 \\ \emptyset & : \text { empty set, } 11 \\ \subseteq & : \text { subset, } 11 \\ \cup & : \text { union, } 12 \\ \mathcal{P}(X) & : \text { power set of } X, 14 \\ (a, b) & : \text { ordered pair, } 14 \\ A \times B & : \text { Cartesian product, } 16 \\ \cap & : \text { intersection, } 17 \\ A \backslash B & : \text { domain of } F, 19 \\ \mathcal{D}(F) & : \text { range of } F, 19 \\ \mathcal{R}(F) & : \text { identity function, } 19 \\ I d_{A} & : \text { inverse function, } 20 \\ F-1 & : \text { composite function, } 20 \\ G \circ F & \end{array}$


$\begin{array}{cl}a R b & : \text { relation, } 21 \\ \varsubsetneqq & : \text { proper subset, } 23 \\ S^{+} & : \text {successor of } S, 24\end{array}$

\section{Chapter 2}

$(\mathbb{N}, 1, \phi)$

IN

$<$

$[1, n]$

$\operatorname{lub}(S), \sup (S)$ $g l b(S), \inf (S)$

$\mathbb{N}_{c}$

$\mathbb{N}_{o}$

$A^{n}$

$(X, \phi)$

$S_{n}$

\section{Chapter 3}

$\boldsymbol{Z}$

$\mathbb{N}_{\mathbb{Z}}$

$\mathbb{Z}$

$\operatorname{gcd}(n, m)$

$(X, \oplus, \odot)$

$\boldsymbol{C}(\mathbb{Z})$

$\mathbb{Z}_{n}$

$\mathcal{P}[X]$

$\mathbb{Z}_{e}$

$\mathcal{P}[\mathbb{Z}]$

$\mathcal{L}(X)$

$(X,+, \cdot,<)$

$1_{X}$

$\mathbb{N}_{X}$

$\mathbb{Z}_{X}$
: natural number system, Peano system, 35

: set of natural numbers, 41

: order on natural numbers, 49

: the set $\{1,2, \ldots, n\}, 53$

: least upper bound of $S \subseteq \mathbb{N}, 55$

: greatest lower bound of $S \subseteq \mathbb{N}, 55$

: even natural numbers, 57

: odd natural numbers, 57

: $n$-fold Cartesian product of $A, 70$

: semigroup, group, 76

: symmetric group of permutations, 77
: the set $\mathbb{N} \times \mathbb{N} / \sim, 85$

: natural numbers in $\mathbb{Z}, 86$

: set of integers, 86

: greatest common divisor of $n$ and $m, 97$

: ring, 106

: set of sequences in $\mathbb{Z}, 106$

: set of integers modulo $n, 107$

: the ring of polynomials over $X, 108$

: ring of even integers, 110

: ring of polynomials over $\mathbb{Z}, 114$

: formal Laurent series over $X, 114$

: ordered integral domain, 116

: identity for multiplication in $X, 117$

: set of natural numbers of $X, 118$

: set of integers of $X, 119$ 


\section{Chapter 4}

$\mathbb{Q}$

$\mathbb{Z}_{\mathbb{Q}}$

$\mathbb{Q}$

$(A, B)$

$|r|$

$\left\{r_{n}\right\}_{n \geq 1}$

$\lim _{n \rightarrow \infty} x_{n}$

$C(\mathbb{Q})$

$\mathbb{Q}_{F}$
: the set of rationals, 133

: integers of $\mathbb{Q}, 136$

: field of rational numbers, 136

: gap in Q, 148

: absolute value of rationals, 152

: sequence of rationals, 153

: limit of the sequence $\left\{x_{n}\right\}, 157$

: set of Cauchy sequences in $\mathbb{Q}, 171$

: fractions of a field $F, 174$

\section{Chapter 5}

$(\alpha, \beta) \quad:$ cut of rationals, 182

$\mathbb{R}$

: set of all cuts, 186

$\alpha_{r}$

$\left\{\alpha_{n}\right\}_{n \geq 1}$

: cut induces by $r, 186$

$\left\{r_{n}\right\}_{n \geq 1} \sim\left\{s_{n}\right\}_{n \geq 1}$ : equivalence of rational sequences, 206

$\left[\left\{r_{n}\right\}\right]$

: equivalence class containing $\left\{r_{n}\right\}_{n \geq 1}, 208$

$\mathbb{R}_{D}$

$\mathbb{R}_{C}$

: real numbers by Dedekind's method, 224

: real numbers by Cantor's method, 225

\section{Chapter 6}

$[x]$

$-S$

$|\alpha|$,

$\mathcal{C}_{\mathbb{R}}$

e

$\pi$
: integral part of $x, 239$

$:\{-s \mid s \in S\}, 240$

: positive $n^{\text {th }}$ root, 244

: absolute value of a real number, 246

: set of all real convergent sequence, 249

: Euler's number, 250

: pi, 253 

$(a, \infty),[a,+\infty)$, $(a, b)[a, b],[a, b)$, $(a, b],(-\infty, b], \quad\}$ : intervals, 261 $(-\infty, b)$, $(-\infty,+\infty)$
$A^{\circ}$
$\lambda(I)$
$A \sim B$
$N_{0}$
c
$2^{\aleph_{0}}$
$\lim _{x \rightarrow u} f(x)$
$f_{1} \wedge f_{2}$
$f \vee f_{2}$
$d_{\alpha}(x, y)$
$d_{1}(x, y)$
$d_{\infty}(x, y)$
$d_{2}(x, y)$
$(\mathbf{x}, \mathbf{y})$
$\rho_{1}(f, g)$
$\rho_{\infty}(f, g)$
$B(x, r)$
$\bar{B}(x, r)$
: closure of a set $A, 264$
: interior of a set $A, 266$
: length of an interval, 273
: equivalence of sets, 280
: aleph-nought, 284
: cardinality of the continuum, 284
: 287
: limit of a function, 298
: maximum of functions, 299
: minimum of functions, 299
: 308
: 309
: uniform metric, 309
: Euclidean metric, 309
: inner product, 309
: $L_{1}$-metric, 311
: 311
: open ball, 313
: closed ball, 313

\section{Chapter 7}

$\begin{array}{ll}\mathbb{C} & : \text { complex numbers, } 322 \\ i & : \text { imaginary unit, } 323 \\ \operatorname{Re}(Z), & : \text { real part of } Z, 325 \\ \operatorname{Im}(z), & : \text { imaginary part of } Z, 325 \\ \frac{|Z|}{Z} & : \text { absolute value of } Z, 328 \\ \mathbb{H} & : \text { conjugate of } Z, 330 \\ & : \text { quaternions, } 350\end{array}$




\section{References}

[Art] Artin, M.: “Algebra", Prentice-Hall Int., Inc., Englewood Cliffs, N.J., 1991.

[Bar] Bartle, R.G. and Sherbert D.R.: "Introduction to Real Analysis" (Second Edition), John Wiley and Sons Inc., New York, 1994.

[Bel] Bell, E.T.: "Men of Mathematics", Simon \& Schuster, Inc. New York, 1965.

[Ber] Berberian, S.K.: "A First Course in Real Analysis", Springer-Verlag, New York, 1994.

[Boa] Boas, Jr., Ralph P.: "A Primer of Real Functions", Mathematical Association of America, 1981.

[Boy] Boyer, Carl B. and Merzback Uta C.: "A History of Mathematics", (2nd Edition) John Wiley and Sons, New York, 1989.

[Bur] Burril, C.W.: "Foundations of Real Numbers", McGraw Hill, Inc., New York, 1951.

[Coh-1] Cohen, L.W. and Eherlich, G.: "Structure of the Real Number System", D. Van Nostrand Company, Inc. Princeton, N.J., 1963. 
[Coh-2] Cohen, P.J.: "Set Theory and the Continuum Hypothesis", W.A. Benjamin, Inc., New York, 1966.

[Con] Conway, John B.: "Functions of One Complex Variable" (Second Edition), Springer-Verlag, New York, 1973.

[Cop-1] Copson, E.T., "Metric Spaces", Cambridge University Press, New York, 1968.

[Cop-2] Copson, E.T.: "Introduction to the Theory of Functions of a Complex Variable", Oxford University Press, New York, 1992.

[Cou] Courrant, R. and Robbins, H.: "What Is Mathematics", Oxford University Press, New York, 1941.

[Dav] Davis, Philip J. and Hersh Reuben: "The Mathematical Experience", Penguin Books Ltd., 1984.

[Die] Dieudonne, J.: "Mathematics - The Music of Reasons", (English translation by H.G. and S.C. Dales), SpringerVerlag, New York, 1987.

[Dra] Drake, F.R. and Singh D.: "Intermediate Set Theory", John Wiley \& Sons, New York, 1996.

[Ebb] Ebbinghaus, H.-D. et.al.: "Numbers", Springer-Verlag, New York, 1990.

[End] Enderton, H.B.: "Elements of Set Theory", Academic Press, New York, 1977.

[Est] Estermann, T.: "On the Fundamental Theorem of Algebra", J. Lond. Math. Soc. (31) 1956, pp. 238-240.

[Eve] Eves, Howard: "An Introduction to the History of Mathematics", (Fourth Edition), holt, rinehart and winston, New York, 1976. 
[Fra] Fraleigh, John B.: "A First Course in Abstract Algebra", Addison-Wesley Publishing Co., Inc., New York, 1987.

[Gau] Gaughan Edward D.: "Introduction to Analysis" (3rd Ed.), Brooks/Cole Publishing Co., California, 1987.

[Gof] Goffman, C. and Pedrick, G.: "First Course in Functional Analysis", Prentice-Hall Int., 1965.

[Hal] Halmos, P.R.: "Naive Set Theory", Springer-Verlag, New York, 1974.

[Hen] Henle, James M.: "An Outline of Set Theory". SpringerVerlag, New York, 1986.

[Kan] Kantor, I.L. and Solodovmikov, A.S.: "Hypercomplex Numbers, An Elementary Introduction to Algebras", Springer-Verlag, New York, 1989.

[Kli] Kline, M.: "Mathematics Thoughts from Ancient to Modern Times", Oxford University Press, New York, 1972.

[Kri] Krishnamurthy, V.: "Cultural Excitement and Relevance of Mathematics", Wiley Eastern Ltd., India, 1990.

[Lan] Landau, E.: "Foundations of Analysis", Chelsea Publishing Co., New York, 1951.

[Lang] Lang, S.: "Algebra" (3rd edition), Addison-Wesley Pub. Co. Inc., New York, 1993.

[Mao] Maor, E.: "To Infinity and Beyond - A Cultural History of the Infinite", Birkhäuser, Boston, 1987.

[Men] Mendelson, E.: "Number Systems and the Foundations of Analysis", Academic Press, New York, 1973. 
[Niv] Niven, I.M.: "Numbers : Rational and Irrational", Random House, New York, 1961.

[Pon] Ponnusamy, S.: "Foundations of Complex Analysis", Narosa Publishers, India, 1995.

[Rem] Remmert, R.: "Theory of Complex Functions". Readings in Mathematics, Springer-Verlag, New York, 1991.

[Rud] Rudin, W.: "Principles of Mathematical Analysis" (3rd Edition), McGraw Hill Inc., 1976.

[Ste] Stewart, Ian and David Tall: "The Foundations of Mathematics". Oxford University Press, 1977.

[Sto] Stoll, R.: "Set Theory and Logic", Dover Publications, New York, 1963.

[Sup] Suppes, P.: "Axiomatic Set Theory", Van Nostrand Reinhold, 1961.

[Tre] Trewin, A.H.: "Mathematics with a Difference", Macmillan of Australia, 1968. 


\section{Index}

A

Abel, Niels Hernik 180

abelian group 76

absolute value, 199, 219

of a complex number 328

of quaternions 350

addition,

of complex numbers 322

of cuts 188

of integers 86

of natural number 42

of polynomials 108

additive inverse 86

additive inverse of a cut 189

amicable 33

Archimedean 138

Archimedean ordered integral domain 176

Archimedes 255

Archimedean property of the reals 239

Argand's inequality 342

Argand, Jean Robert 320

argument 329

associative $43,45,75$

atomic formulae 9

axiom, 7

of choice 26,74

of comprehension 15

of empty set 10

of extensionality 10

of pairing 11

of power set 14

of regularity 27

of replacement 25

of separation 15

of union 12

B

base 2, 104

Bernays, Paul 28

Bernoulli, Daniell 233

Bhaskara 255

bijective 19

binary,

expansion 259

operation 42,75

representation 104

Binomial,

coefficients 141

inequality 141

theorem 141

Bolzano, Bernard 2, 23, 57, 130, 234

Bolzano-Weierstrass property 202, 245

Bombelli, Rafael 319

Borel, Emile 235 
bounded,

above 54,72

below 55,72

sequence 200

set 264,298

Brahmagupta 81

C

cancellation law, for addition 44 for multiplication 47,51 in integral domains 109

Cantor-Bernstein-Schröder theorem 282

Cantor's ternary set 260

Cantor, Georg 2, 234

Cardano, Geronimo 319

cardinal numbers 284

cardinality,

aleph-nought 284

of a set 283

of power set 286

of the continuum 284

Cartesian product 17

Cartesian product, $n$-fold 70

Cauchy,

complete ordered field 178

completeness 202

sequence 171,219

Cauchy's,

inequality 309,334

minimum theorem 348

Cauchy, Augustin Louis 180, 234, 236

Cayley numbers 351

Cayley, Arthur 351

center 314 characteristic, 119

zero 119

choice function 26

Chung-chi, Tsu 255

closed,

ball 314

interval 261

set 262

closure 264

codomain 19

coefficient leading 108

coefficients 108

Cohen, Paul 3

common divisor 97

commutative, 45

binary operation 75

group 76

law 43

semigroup 76

compact set 269,316

complete,

ordered field 231

metric space 312

completeness, axiom of 238

complex numbers 320,324

composite 20, 96

congruent modulo 107

conjugate 330

connected 291

constant, 8

function 297,317

sequence 162,208

term 108

continuous, 296,317

at a point 296

everywhere 317

continuum 284 
Continuum Hypothesis 285

convergent sequence 158,220

countable 57

countably finite 57

Cousin, Pierre 235

cross product 351

cut 182,185

induced 186

bigger than 195

rational 186

smaller than 195

D

d'Alembert, Jean le 233

De Moivre's formula 330

decimal, 151, 256

rational 152

representation $104,149,256$

periodic 256

Dedekind, Richard 33180

degree 108

denseness of irrationals 199

denseness of rationals 199, 218

Descartes, René 81

Diophantos 319

Dirichlet, Peter Gustav Lejeune 180,234

disconnected 291

discontinuity 296

distributive, 45

property 45

division,

algorithm 93

ring 348

domain 19,21

dot product 351
E

empty,

function 19

set 11

equivalence,

class 22

relation 21

equivalent $84,206,281$

Eudoxus 130

Euler's number 250

Euler, Leonhard 233, 320

even number 57,101

Existence of $\mathbb{N} 36$

exponentiation, 47,48

laws of 47

F

factor 96

factorial 250

field, 111

of fractions 174

of rationals 137

ordered 173

finite,

cardinals 284

set 23

formula 9

Fourier, Joseph 234

fractional part 140

Fraenkel, Abraham A. 3

Frege 33

Frobenius, Georg 351

function, 19

constant 297,317

successor 34

fundamental theorem of algebra 320,343 
G

Gödel, Kurt 3, 29

gap 148

Gauss, Carl Friedrich 320, 321

General Principle of Induction 67

Girard, Albert 319

greater than, 49 or equal 50

greatest, common divisor 97

lower bound 55,72

lower bound property 74

group, 76

abelian 76

commutative 76

H

Hamilton, Sir William Rowan 320, $321,347,348$

Hankel, Hermann 130

Harriot, Thomas 319

Heine, Eduard 234

Heine-Borel theorem 271

Hermite, Charles 255

Hippasus 124

hyper-complex numbers 350

I

identity, 86

for multiplication 44

function 19

imaginary,

axis 327

part 325

unit 324

index 278

indicator function 298
Induction,

General Principle 67

Modified Principle 67

Principle of Mathematical 35

inductively 40

infinite,

cardinals 285

set 23

injective 19

integer system 87

integers $88,119,173$

integral,

domain 92, 109

part 140,239

interior point 266,314

Intermediate value theorem 302

intersection 17

interval, 260

closed 261

open 261

inverse 75

irrational number 198, 244

irrational points 147

irreflexive 21

isomorphic, 79

fields 120

integral domains 120

ordered field 120

ordered integral domain 120 isomorphism of,

field 120

groups 79

integral domain 120

ordered field 120

ordered integral domain 120

semigroups 79 
$\mathbf{J}$

Jordan, Camilla 235

\section{$\mathbf{K}$}

Kronecker, Leopold 82, 181

Kummer, Ernst Eduard 181

L

$L_{1}$-metric 311

Lagrange 236

Lambert, Johann Heinrich 255

Laplace 236

larger than 93

Laurent series 114

leading coefficient 108,114

least upper bound, 55,72

$$
\text { property } 74
$$

Lebesgue, Henri 237

left-distributive 78

left-end point 260

Leibniz, Gottfried Wilhelm 320

length 310

less than 49,138

less than or equal 50

limit,

of a function 298

of a sequence 158

point 266

Lindermann, Ferdinand 255

linear,

order 23,73

polynomials 108

linearly ordered set 73

lower bound 55,72

lub-property 238
M

magnitude 328

Mahaviracharya 81

maximum, 72, 294

value 294

member 7

metric, 308

space 308

$L_{1} 311$

uniform 311

complete 312

minimum, 72, 294

value 294

Modified Principle of Induction 67

monotonically,

decreasing 201

increasing 201

multiplication of,

complex numbers 322

integers 89

of natural numbers 47

of polynomials 108

N

natural number system 35

natural numbers, 34,41

of an integral domain 118

negative, 138

cut 189

integers 88

sequence 214

neighborhood 305

Nested interval property 274

nested intervals 273

Neumann, von 28

Newton, Isaac 320 
nonempty set 16

norm 310

null sequence 207

number,

even 101

real algebraic 245

composite 96

irrational 199

odd 97

rational 198

prime 96

purely imaginary 325

rational 133

transcendental 255

numbers,

perfect 33

real 207,225

complex 320

natural 34,41

perfect 33

O

odd number 57,97

Ohm, Martin 130

one 34

one-one 19

onto 19

open,

ball 314

cover 270

interval 261

set 267

order, 23,73

complete $75,178,230$

linear 23, 73

partial 23

total 23,73 ordered, n-tuple 71

field 173

integral domain 113

pair 14

set 73

subfield 173

triple 71

$\mathbf{P}$

parallelogram identity 333

partition 291

partial order 23

partially ordered 23

Peano system 35

Peano, Giuseppe 33

perfect numbers 33

periodic decimal 151, 256

permutation 77

pi 253

Pigeonhole principle 66

Poincaré, Henri 235

point of discontinuity 296

polar form 329

polynomial 108

positive, 115, 137

$n^{\text {th }}$ root 244

cut 189

integers 88

sequence 214

power set 14

prime,

decomposition 100 number 96

primitive 7

Principle of Mathematical Induction 35 
product of,

cuts 189

natural numbers 44

proper subset 23

propositional connectives 8

purely imaginary number 325

Pythagoras 32

Pythagorean triplets 33

$\mathbf{Q}$

quadratic polynomials 108

quantifiers 8

quaternions 350

quotient 95

$\mathbf{R}$

radius 314

range 19,21

rational,

cut 186

number 133,198

real,

algebraic number 245

axis 327

line 260

number 225

numbers 207

part 325

recursively 40

reflexive 21

relation 21

relative complement 18

relatively prime 99

remainder 95

Riemann, Bernhard 234

right,

distributive 78 end point 260

ring, 92, 106

division 348

of integers modulo $n 107$

of polynomials 109

with identity 106

Roots of real polynomials 304 Russell, Bertrand 3

S

scalar,

part 351

product 351

semigroup 76

separated 290

separation 291

sequence, $66,107,153$

Cauchy 171, 219

constant 162

convergent 158,220

negative 214

null 207

positive 214

set, 7

compact 316

bounded 264

closed 262

infinite 23

linearly ordered 73

open 267

ordered 73

singleton 11

totally ordered 73

compact 269

well ordered 73

countable 57

countably finite 57 
finite 23

linearly ordered 23

partially ordered 23

successor 24

singleton set 11

skew field 348

Skolem, T. 3

smaller than 93

Steinitz, Ernst 130

Stifel, M. 180

Stolz, Otto 180

subdomain 120

subfield, 173

ordered 173

subsequence 201

subset, 11

proper 23

successor, 24

function 34

sum of cuts 188

surjective 19

symmetric, 21

difference 107

group of permutations 77

symmetry 308

system,

natural number 35

Peano 35

$\mathbf{T}$

tens place 104

total order 23,73

totally ordered set 73

transcendental number 255

transitive 21

triangle-inequality $152,199,308$,

333 trichotomy law 50

$\mathbf{U}$

uncountable set 57

uniform metric 311

union 12

units place 104

upper bound 54,72

V

variable 8,108

vector part 351

vector product 351

W

Wallis, John 130

Weber, Heinrich 181

Weierstrass, Karl Theodore 130, 234,235

well-ordered set 73

well-ordering, 55

of $\mathbb{N} 56$

principle 74

Wessel, Caspar 320

Whitehead, Alfred North 3

$\mathbf{Z}$

Zermelo, Ernst 3

zero, 88

divisors 92 